\title{
Radio Interferometric Angle of Arrival Estimation
}

\author{
Isaac Amundson, Janos Sallai, Xenofon Koutsoukos, and Akos Ledeczi \\ Institute for Software Integrated Systems (ISIS) \\ Department of Electrical Engineering and Computer Science \\ Vanderbilt University \\ Nashville, TN 37235, USA \\ isaac . amundson@vanderbilt.edu
}

\begin{abstract}
Several localization algorithms exist for wireless sensor networks that use angle of arrival measurements to estimate node position. However, there are limited options for actually obtaining the angle of arrival using resource-constrained devices. In this paper, we describe a radio interferometric technique for determining bearings from an anchor node to any number of target nodes at unknown positions. The underlying idea is to group three of the four nodes that participate in a typical radio interferometric measurement together to form an antenna array. Two of the nodes transmit pure sinusoids at close frequencies that interfere to generate a low-frequency beat signal. The phase difference of the measured signal between the third array node and the target node constrains the position of the latter to a hyperbola. The bearing of the node can be estimated by the asymptote of the hyperbola. The bearing estimation is carried out by the node itself, hence the method is distributed, scalable and fast. Furthermore, this technique does not require modification of the mote hardware because it relies only on the radio. Experimental results demonstrate that our approach can estimate node bearings with an accuracy of approximately $3^{\circ}$ in $0.5 \mathrm{sec}$.
\end{abstract}

\section{Introduction}

Spatial coordination in wireless sensor networks (WSNs) has received a lot of attention in recent years. In typical solutions, one or more nodes emit a signal, and some property of that signal (e.g. angle of arrival (AOA), time of arrival (TOA), received signal strength (RSS), etc.) is measured and used to derive bearing or range. Angulation or lateration techniques can then respectively be used to estimate a node's position.

Although several techniques exist for determining node position based on bearing information [1], [2], [3], [4], [5], there are few options for actually measuring signal AOA in WSNs. Currently available methods for bearing estimation require a heavy-weight infrastructure [6], rotating hardware [7], [8], directional antennas [9], and/or expensive and sophisticated sensors [10]. Furthermore, such techniques typically require participating nodes to be stationary for extended periods of time. These constraints are often undesirable for WSN deployments, in 
which node size and cost must be kept to a minimum. An AOA approach that does not require additional hardware, runs on the nodes themselves, and is fast enough to support tracking in addition to static localization would be a major step forward.

In this paper, we propose a novel AOA approach for WSNs that uses radio interferometry [11]. The basic idea is to group together three of the four nodes involved in a typical radio interferometric measurement to form an antenna array, which acts as an anchor node. Two transmitters and one receiver are arranged in such a manner that their antennas are mutually orthogonal to minimize parasitic antenna effects (see Figure 1.) The measured phase difference between the receiver in the array and a target node constrains the location of the latter to a hyperbola. The bearing of the target node can then be estimated by computing the angle of the hyperbola asymptote, assuming the target node is not too close to the array.

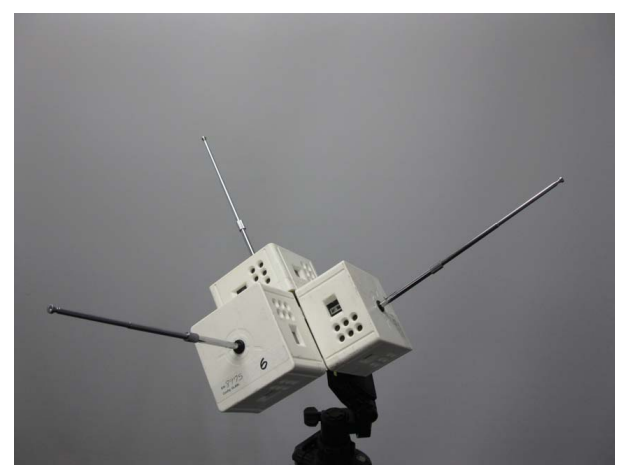

Fig. 1. Antenna array implementation using three XSM motes.

We present several new contributions for estimating the angle of arrival in wireless sensor networks.

1. We describe an RF-based technique for determining target node bearing.

2. We provide a detailed analysis that shows our bearing estimation algorithm is robust to measurement noise and approximation error.

3. We design a real-world implementation using COTS sensor nodes, in which bearing estimation is performed entirely on the resource-constrained motes.

4. We present experimental results that show our approach can rapidly and accurately estimate node bearing.

The remainder of this paper is organized as follows. In Section 2, we discuss other angle of arrival techniques for WSNs. Section 3 describes our proposed system, followed by an error analysis in Section 4. In Section 5, we describe our implementation on a real-world WSN platform. In Section 6, we evaluate our system based on experimental results. Section 7 concludes. 


\section{Related Work}

The RF method we use for determining AOA is based on radio interferometry. The Radio Interferometric Positioning System (RIPS) provides accurate RFbased localization in WSNs [11]. The main idea is that the resource-constrained nodes cannot sample a pure RF signal fast enough, but can process the lowerfrequency envelope of the beat signal that results from the interference of two high-frequency signals. The difference in signal phase measured by two other nodes is a linear combination of the distances between the transmitters and receivers, modulo the wavelength, and can be used for localizing all participating nodes by solving an optimization problem. Although RIPS has centimeteraccuracy and can support inter-node distances of greater than twice the communication range, it requires centralized processing, suffers from high latency, and involves sampling at several frequencies.

A broad spectrum of acoustic beamforming techniques have been proposed to find the angle of incidence of a signal at an array of sensors. The most common techniques include delay-and-sum beamforming, Capon beamforming [12], MUSIC [13], ESPRIT [14] and min-norm [15] algorithms. Since the time of flight of the signal from the source to sensors in the array varies based on their pairwise distances, sensors receive the signal with different phases. While all of these methods compute the bearing of the source from the data streams sampled at the individual sensors, they differ greatly with respect to their angular resolution as well as their computational requirements. In WSNs, angular resolution is typically within $10^{\circ}[16]$.

The Cricket Compass [17] is a device which uses ultrasound to determine orientation with respect to a number of ceiling-mounted beacons. Two receivers are mounted a few centimeters apart on a portable device, and the phase difference of the ultrasonic signal is measured to determine bearing. Although both the Cricket Compass and our approach measure signal phase difference to derive AOA, the two systems use different hardware, signal modalities, phase disambiguation techniques, and bearing derivation algorithms. The Cricket Compass has an accuracy of between $3^{\circ}$ and $5^{\circ}$, depending on the orientation of the compass.

Angle of arrival can be used in different ways for spatial coordination. Triangulation, for example, is the process of determining the position of an object from the bearings of known reference positions. Two such reference positions (or three non-collinear ones in degenerate cases) are enough to localize any number of nodes within range. In [2], a method is given to determine position based on the angular separation (the difference in bearings) between beacons. Other angle of arrival positioning approaches have been developed, including multiangulation using subspace methods [4], anchor bearing propagation [1], and semidefinite programming [3]. Bearing estimates can also be useful when anchor positions are unknown. In [18] and [19], mobile robot navigation methods are presented for arriving at a target position by only observing angular separation between two pairs of landmarks. 


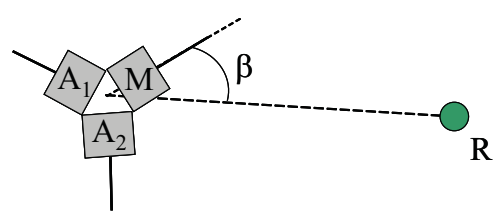

Fig. 2. Array containing a master node $(\mathrm{M})$ and two assistant nodes $\left(A_{1}, A_{2}\right)$. A target node $(\mathrm{R})$ computes its bearing $(\beta)$ from the array.

\section{System Overview}

Radio Interferometric Measurements. Our system consists of a stationary antenna array and cooperating wireless sensor nodes at unknown positions. We assume that the position of the midpoint of the array is known, as well as the distance between the antennas in the array. The array contains three nodes, a master $(\mathrm{M})$ and two assistants $\left(A_{1}, A_{2}\right)$, as shown in Figure 2. At a predetermined time, the master, $M$, and one of the assistants, $A_{1}$, transmit a pure sinusoidal signal at slightly different frequencies, which interfere to create a low-frequency beat signal whose phase is measured by the other assistant in the array, $A_{2}$, and a receiver node, $R$, at an unknown position. Such a measurement is termed a radio interferometric measurement (RIM).

The difference in phase, $\Delta \varphi=\varphi_{R}-\varphi_{A_{2}}$, measured by receiver nodes $R$ and $A_{2}$ is a linear combination of the distances between the transmitters and receivers,

$$
\Delta \varphi=\frac{2 \pi}{\lambda}\left(d_{M A_{2}}-d_{A_{1} A_{2}}+d_{A_{1} R}-d_{M R}\right)(\bmod 2 \pi),
$$

where $\lambda$ is the wavelength of the carrier frequency, $d_{M R}$ is the distance between the master node and target receiver node, $d_{A_{1} R}$ is the distance between the assistant transmitter and the target receiver node, and $d_{M A_{1}}, d_{M A_{2}}$, and $d_{A_{1} A_{2}}$ are the respective distances between all pairs of nodes in the array. Note that the nodes in the array are equidistant from each other, and therefore $d_{M A_{2}}-d_{A_{1} A_{2}}=0$, so the phase difference can be simplified:

$$
\Delta \varphi=\frac{2 \pi}{\lambda}\left(d_{A_{1} R}-d_{M R}\right)(\bmod 2 \pi) .
$$

We denote the distance difference $d_{A_{1} R}-d_{M R}$ by $d_{A_{1} M R}$ and refer to it as a $t$-range. From Equation (1), we can see that if $-\frac{\lambda}{2}<d_{A_{1} M R}<\frac{\lambda}{2}$, the phase difference will fall in the interval $(-\pi, \pi)$. When this is not the case, the possible range of $\Delta \varphi$ will exceed $2 \pi$, which results in a modulo $2 \pi$ phase ambiguity. To avoid this, we would like the maximum possible distance difference to be less than $\frac{\lambda}{2}$. The maximum distance difference will occur when the receiver node is collinear with the transmitters $M$ and $A_{1} \cdot d_{A_{1} M R}$ then corresponds to the 
distance between the master and assistant. Therefore, to eliminate the modulo $2 \pi$ phase ambiguity, we require the distance between antennas in the array to be less than half the wavelength of the carrier frequency.

Having removed the modulo operator, we can rearrange Equation (1) so that known values are on the right hand side.

$$
d_{A_{1} M R}=\frac{\Delta \varphi \lambda}{2 \pi}
$$

The t-range $d_{A_{1} M R}$ defines an arm of a hyperbola that intersects the position of node $R$, and whose asymptote passes through the midpoint of the line $\overline{A_{1} M}$, connecting the master and assistant nodes. Figure 3 illustrates such a hyperbola with foci $A_{1}$ and $M$. The absolute value of the distance differences between the foci and any point on a hyperbolic arm is constant, formally defined as

$$
\frac{x^{2}}{a^{2}}-\frac{y^{2}}{b^{2}}=1
$$

where $(x, y)$ are the coordinates of a point on the hyperbola, $a$ is the distance between the hyperbola center and the intersection $H$ of the hyperbola with the axis connecting the two foci, and $b$ is the length of the line segment, perpendicular to the axis connecting the foci, that extends from $H$ to the asymptote.

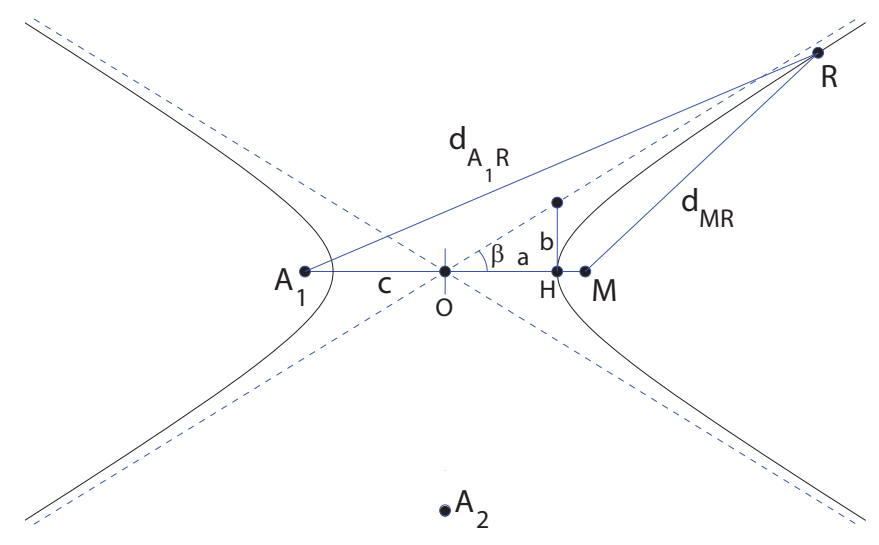

Fig. 3. The t-range defines a hyperbola that intersects node R, and whose asymptote passes through the midpoint of the two transmitters in the array.

Bearing Approximation. The hyperbola in Figure 3 is centered at $O$, and the distance between $O$ and either focus is denoted by $c$. Furthermore, it can be shown that $c^{2}=a^{2}+b^{2}$ [20]. From the figure, we see that the bearing of 
the asymptote is $\beta=\tan ^{-1}\left(\frac{b}{a}\right)$. Therefore, in order to solve for $\beta$, we must determine the values of $b$ and $a$.

We can solve for $a$ by observing that

$$
d_{A_{1} R}-d_{M R}=d_{A_{1} H}-d_{M H}
$$

because, by definition, the distance differences between the foci and all points on the hyperbola are constant. From Figure 3, we see that we can substitute $(c+a)$ for $d_{A_{1} H}$ and $(c-a)$ for $d_{M H}$, and therefore,

$$
d_{A_{1} R}-d_{M R}=(c+a)-(c-a)=2 a .
$$

From Equation (2), we know the value of $d_{A_{1} R}-d_{M R}$, which is the t-range, and therefore $a=\frac{d_{A_{1} M R}}{2}$. We can then solve for $b$, using $b=\sqrt{c^{2}-a^{2}}$. In terms of known distances, the bearing of the asymptote is then defined as

$$
\beta=\tan ^{-1}\left(\frac{\sqrt{\left(\frac{d_{A_{1} M}}{2}\right)^{2}-\left(\frac{d_{A_{1} M R}}{2}\right)^{2}}}{\left(\frac{d_{A_{1} M R}}{2}\right)}\right) .
$$

In Figure 3, we see the case where $d_{A_{1} M R}>0$, and the position of $R$ lies on the right arm of the hyperbola. If the phase difference is negative (i.e., $\varphi_{R}<\varphi_{A_{2}}$ ) then the position of $R$ will lie on the left arm of the hyperbola. When this is the case, $\beta$ is taken clockwise, and we must adjust it by subtracting it from $\pi$.

The line $\overline{A_{1} M}$ connecting the two foci is called the transverse axis of the hyperbola, and is a line of symmetry. This implies that although we know $b$, we do not know its sign, because mirrored positions on either side of the transverse axis will result in the same $d_{A_{1} M R}$. Therefore, the asymptote bearing $\beta$ we obtained using this method could be either positive or negative. To find which bearing is correct, we can switch the roles of the assistant nodes in the array and perform another RIM. This will generate a different t-range, and hence another hyperbolic arm with foci $A_{2}$ and $M$.

Each hyperbola provides us with two angles $\pm \beta_{i}$, where $\beta_{i}$ is the angle of the asymptote with the transverse axis, $\overline{A_{i} M}$. Of course, these angles will be offset from the global $x$-axis, because the orientation of $\overline{A_{i} M}$ may not be 0 . Adjusting for this, one of the $\beta_{1}$ bearings, and one of the $\beta_{2}$ bearings will point in the same direction, which will approximate the actual bearing of $R$, as illustrated in Figure 4. Due to the position difference between the centers of the two hyperbolas, we do not expect these two angles to be equal, therefore we define a small threshold $\epsilon_{\beta}$, such that if $\left|\beta_{1}-\beta_{2}\right|<\epsilon_{\beta}$, these two angles are considered a match. We then take the average of the two angles to obtain our bearing estimate, $\hat{\beta}$.

Because points on the hyperbola converge with the asymptote as their distance from the hyperbola center increases, the bearing approximation error is larger when $R$ is close to the array. We therefore make the assumption that node $R$ is a sufficient distance from the array. In Section 4 , we show that this distance does not need to be large when using small-aperture arrays. 


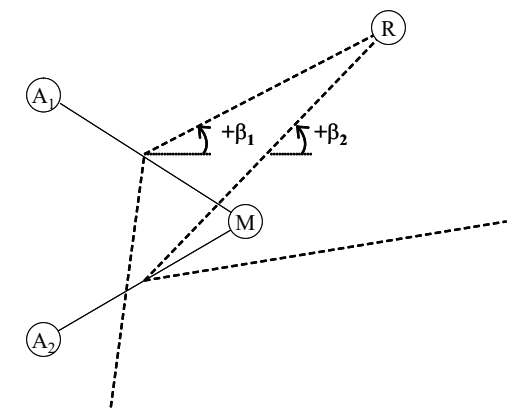

Fig. 4. Determining the true bearing of $R$ is accomplished by selecting $+\beta$ or $-\beta$ from each master-assistant pair, such that the difference between the two angles is below the threshold $\epsilon_{\beta}$.

\section{Error Analysis}

In this section, we present an error analysis of the proposed bearing estimation technique. It is important to note that, although we use phase differences as input to our bearing estimation algorithm, the algorithm is generalizable to smallaperture sensor arrays that can derive distance differences using any means. For instance, RF ultra wide band antenna arrays, acoustic or ultrasonic sensors, and other types of arrays that can yield time-difference-of-arrival (TDOA) measurements from (sufficiently) distant sources fall into this category. Therefore, in this section, we assume the inputs to be distance differences. Notice that the distance differences are linearly related to RIM measurements (see Equation (2)), and therefore the error sensitivity results presented below remain valid. In the generalized case, the same applies to TDOA measurements, from which the distance differences can be computed via multiplication of the respective signal propagation speed (speed of sound for acoustic, speed of light for RF).

Typically, bearings are computed from distance differences by solving a nonlinear set of equations using iterative techniques. Such techniques are prohibitive on low-end microprocessors due to their computational cost. We make a set of assumptions that allows us to compute bearing estimates in a reasonably simple way. While our bearing estimation technique is computationally less expensive than traditional nonlinear optimization techniques, our simplifying assumptions introduce estimation errors, which we identify below.

- Measurement noise. The distance differences observed by the receiver nodes contain measurement noise. The measurement noise can be attributed to, for instance, non-ideal signal propagation, noise from the electrical circuitry of the receiver, sampling error and quantization error of the analog-to-digital converter (ADC).

- Asymptote approximation. For a pair of transmitters, we approximate the bearing of the receiver with the angles of the asymptotes of the hyperbola. 
This is a good approximation if the receiver is sufficiently far from the transmitters, because the hyperbola converges on its asymptote. However, for close receivers, errors due to this assumption will not be negligible.

- Translation of bearing candidates. At least two transmitter pairs are required to unambiguously compute the bearing because, for just one transmitter pair, the angles of both asymptotes are possible solutions. We refer to the two solutions as bearing candidates. Since, for a transmitter pair, we compute the bearing candidates with respect to the midpoint of the segment defined by the two antennas, fusing bearing candidates from two different transmitter pairs is not possible without knowing the distance of the receiver. We use the far-field assumption (i.e., that the receiver is infinitely far from the transmitter array) to carry out the disambiguation and fusion of bearings, introducing an error this way.

We intentionally omit the analysis of array position and orientation errors and instead make the following assumptions:

- Antenna configuration is known. The transmitter locations are assumed to be given. It is assumed that the transmitter nodes are fabricated with a prescribed antenna separation.

- Relative bearings. We assume that the computed bearings are given in the local coordinate system of the array. Hence, the location and orientation errors of the array are not considered in the error analysis of the bearing estimation.

We first analyze the sensitivity of the bearing estimates to noise in the distance difference inputs. Second, we analytically derive the errors related to the asymptote approximation and to the translation of bearing candidates. These errors depend on the bearing and distance of the target receiver, relative to the transmitter array. Finally, we provide an analysis of the total bearing estimation error resulting from both noise in the inputs and the errors due to the asymptote approximation and the translation of bearing candidates.

Sensitivity of bearing to measurement noise. A distance difference from a pair of transmitters in the array constrains the location of the receiver to one arm of a hyperbola, the foci of which are the positions of the two transmitters. For the sake of simplicity, let us assume that the two transmitters $M$ and $A_{1}$ are located at $(c, 0)$ and $(-c, 0)$, respectively (see Figure 3$)$. If the measured distance difference is positive, the receiver is constrained to the right arm of the hyperbola, while if the distance difference is negative, the receiver is located on the left arm. We approximate the bearing of the receiver using the asymptote angles as follows:

$$
\hat{\beta}= \begin{cases} \pm \tan ^{-1}\left(\frac{\sqrt{c^{2}-a^{2}}}{a}\right), & \text { if } a>0 \\ \pm \frac{\pi}{2}, & \text { if } a=0 \\ \pi \mp \tan ^{-1}\left(\frac{\sqrt{c^{2}-a^{2}}}{a}\right), & \text { if } a<0\end{cases}
$$


We analyze the sensitivity of the bearing estimates $\hat{\beta}$ to noise in the distance difference by taking the partial derivative of Equation (4) with respect to the distance difference. To see what amplification effect an error in a given distance difference $d$ produces on the bearing estimate, we need to evaluate the partial derivative at $d$.

Figure 5a shows the relation between the measured distance difference $d$ and the bearing candidates $\hat{\beta}$ when the antenna separation is half the wavelength $\left(\frac{\lambda}{2}\right)$. Notice the ambiguity of the bearing candidates. Figure $5 \mathrm{~b}$ plots $\frac{\delta \hat{\beta}}{\delta d}$ for each solution of $\hat{\beta}$. This figure shows that when the absolute value of the measured distance difference is close to the antenna separation, the computed bearing candidates are very sensitive to measurement noise. For instance, if the distance difference is $80 \%$ of the antenna separation, an infinitesimally small error in the measurement will be amplified ten-fold in the bearing estimate.

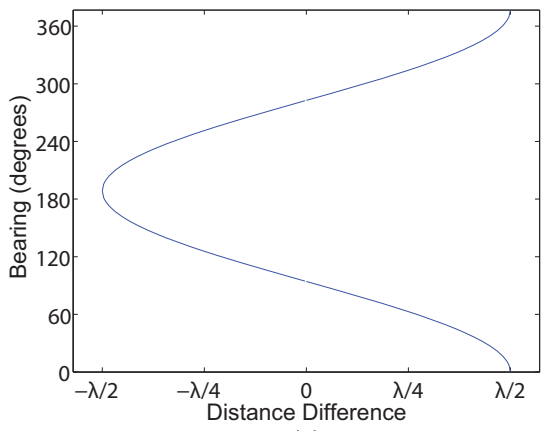

(a)

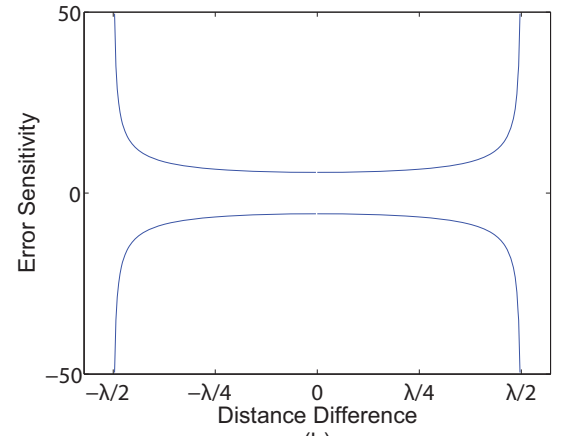

(b)

Fig. 5. (a) Relationship between measured distance difference and computed bearing. (b) Sensitivity of the computed bearing to measurement noise.

Asymptote approximation. The error of approximating a hyperbola with its asymptote is the difference between the approximated bearing $\hat{\beta}$ and the actual bearing $\beta$ of the receiver. Assuming that the receiver $R$ is located at $(u, v)$, the actual bearing will be $\beta=\tan ^{-1}\left(\frac{v}{u}\right)$. Hence, the error $\epsilon$ introduced by the asymptote assumption is

$$
\epsilon=\hat{\beta}-\beta= \begin{cases} \pm \tan ^{-1}\left(\frac{v}{u}\right) \mp \tan ^{-1}\left(\frac{\sqrt{c^{2}-a^{2}}}{a}\right), & \text { if } a>0 \\ 0, & \text { if } a=0 \\ \mp \tan ^{-1}\left(\frac{v}{u}\right) \pm \tan ^{-1}\left(\frac{\sqrt{c^{2}-a^{2}}}{a}\right), & \text { if } a<0\end{cases}
$$

Figure 6a shows the error introduced by the asymptote approximation when the receiver is located respectively one, two, and three times the antenna distance away from the midpoint of the segment connecting the two antennas. As 
expected, the error of the approximation decreases as the distance of the receiver from the transmitter array increases, that is, as the hyperbola converges on its asymptote. As we can see, the maximum error introduced by the asymptote assumption is less than $0.6^{\circ}$, as little as three antenna distances away.

Translation of bearing candidates. For a pair of transmitter antennas, it is not possible to unambiguously approximate the bearing of the asymptote. Because the hyperbola arm has two asymptotes, the angle of either one can be the correct bearing estimate. Hence, we need two transmitter antenna pairs for disambiguation. Let us treat the bearing candidates (computed from the t-ranges of both transmitter antenna pairs) as vectors of unit length, with bases at the center of the hyperbolas, and whose angles are the computed bearing candidates. Since these vectors are given in the coordinate system of the respective hyperbolas, we need to transform them to the coordinate system of the array. This transformation includes a translation and a rotation. Then, we translate each vector such that its base is at the origin. Clearly, the bearing vector translated this way will not point directly toward the target receiver anymore, but if the receiver is sufficiently far from the transmitter array, the introduced angular error will be small. Finally, we disambiguate the bearing candidates by finding two that have approximately the same value.

Let us now express the angular error caused by the translation of bearing candidates. We assume that the transmitter is a uniform circular array of three antennas, with pairwise antenna distance of $\frac{\lambda}{2}$. The coordinate system of the array is set up such that the midpoint of the array is at the origin, and antenna $M$ lies on the positive side of the $x$-axis. Let us consider only the correct bearing candidate (the other will be discarded later) for transmitter pair $M$ and $A_{1}$. Furthermore, let us assume for now that the bearing candidate has no error. The difference between the actual bearing of the target receiver and the angle of the bearing candidate translated to the origin gives the angular error of the far-field assumption.

Figure $6 \mathrm{~b}$ shows the error introduced by the far-field assumption when the receiver is located respectively one, two, and three times the antenna distance away from the midpoint of the segment connecting the two antennas. As we can see, as few as three antenna distances away, the maximum error introduced by the antenna assumption is less than $5^{\circ}$. In this particular antenna arrangement, the maximum errors are at $15^{\circ}$ and $225^{\circ}$, respectively.

Compound bearing estimation error. Since one transmitter pair reports two bearing candidates, at least two transmitter pairs are required to resolve this ambiguity. For the sake of simplicity, let us assume that we have two transmitter pairs. Clearly, there must be one bearing candidate for each transmitter pair that is close to the true bearing. Except for some degenerate cases, the other two bearing candidates will be significantly different than the true bearing, and will not be close to each other (see Figure 4). Therefore, in order to disambiguate between the correct and incorrect bearing candidates, we take all possible pairs 


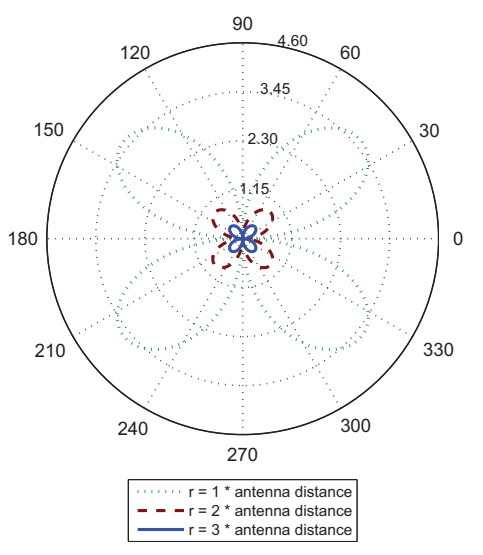

(a)

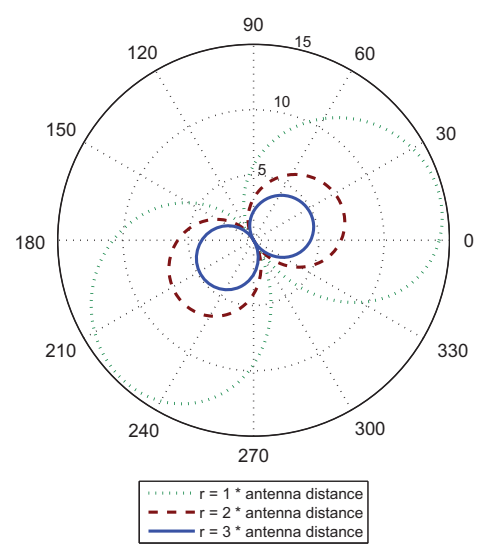

(b)

Fig. 6. Error in bearing (in degrees) caused by (a) the assumption that the receiver lies on the asymptote, and (b) assuming that bearing from the midpoint of the segment connecting the two antennas equals the bearing from the origin of the array coordinate system.

of bearing candidates, one from the first transmitter pair and the other from the second transmitter pair, and find the pair with the least pairwise angular difference. The reported bearing estimate is computed as the average of the two closest bearing candidates.

Figure 7 shows the bearing estimation errors considering the above three types of error sources, averaged over 500 simulation rounds. We added a Gaussian noise to the distance differences, with mean zero and standard deviation set to $5 \%$ of the antenna distance. The plot suggests that the expected bearing estimation errors are below $5^{\circ}$, and peak around $30^{\circ}, 150^{\circ}, 240^{\circ}$ and $330^{\circ}$, exactly where the individual transmitter pairs exhibit high error sensitivity.

\section{Implementation}

Our system is implemented using Crossbow ExScal motes (XSMs) [21], which use the Texas Instruments CC1000 radio chip and transmit in the $433 \mathrm{MHz}$ range. Three XSMs form the array. Because the two transmitting antennas are close to each other, they will suffer from parasitic effects [22]. To minimize this negative interference, we place the nodes in a mutually orthogonal configuration. All sensor nodes are elevated approximately 1.5 meters to reduce ground-based reflections. The antenna array is pictured in Figure 1. All nodes in our system execute the same distributed application, coded in nesC, and run the TinyOS

operating system. All operations run locally, and there is no offline or PC-based 


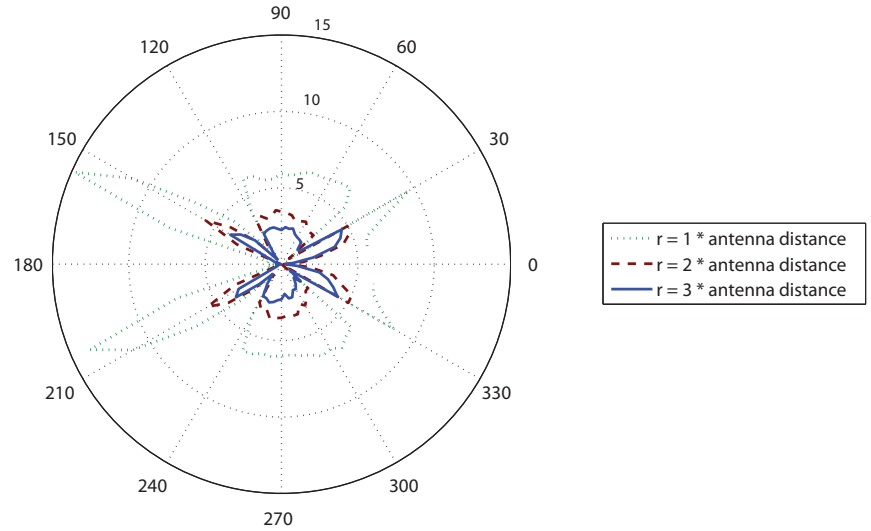

Fig. 7. Absolute error of bearing estimation (in degrees) caused by noisy distance differences, averaged over 500 simulation rounds. The standard deviation of the distance difference errors is $5 \%$ of the antenna distance.

processing involved. The entire application requires 3 kilobytes of RAM and 55 kilobytes of program memory (ROM).

Run-time. Figure 8 is a sequence diagram of the system run-time components using a setup of one array and a single target receiver node. Because phase difference is used to determine bearing, each node must measure the signal phase at the same time instant. This requires synchronization with accuracy on the order of microseconds or better. A SyncEvent message [23] is broadcast by the master transmitter, and contains a time in the future for all participating nodes to start the first RIM. Each array then performs two RIMs, one for each masterassistant pair. Signal transmission involves acquiring and calibrating the radio, transmitting the signal, then restoring the radio to enable data communication. The assistant nodes in the array store their phase measurements until both master-assistant pairs have finished their RIMs, at which point they broadcast their phase measurements to the target nodes. The target nodes then calculate their bearings from the array.

\section{Evaluation}

To evaluate the accuracy of our system, we perform two experiments. In Experiment 1 , we measure the bearing accuracy of six receiver nodes, which are evenly spaced around the array every $60^{\circ}$ at a distance of ten meters from the array center. This experiment demonstrates how the bearing error changes with respect to array orientation. In Experiment 2, we measure the bearing accuracy 


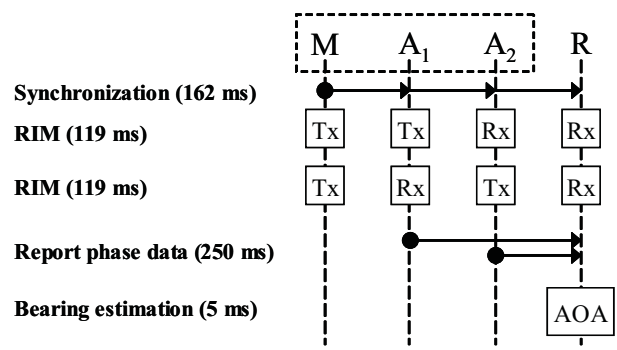

Fig. 8. Sequence diagram of RIM schedule with one array (dotted box) and the target receiver node $(\mathrm{R})$.

of 14 receiver nodes from three arrays surrounding the sensing region in an outdoor, low-multipath environment. This experiment is more representative of a real-world deployment with multiple anchors. Figure 9 illustrates our setup for the two experiments.

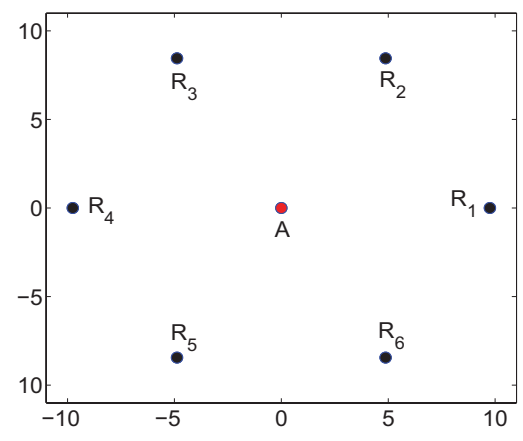

(a)

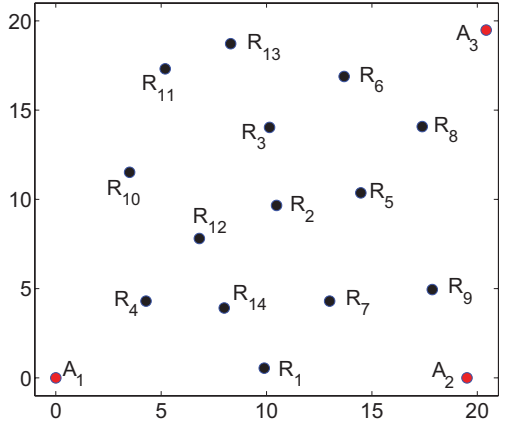

(b)

Fig. 9. Experimental setup. (a) Experiment 1. Bearing accuracy of one array. Six receiver nodes $\left(R_{1} \ldots R_{6}\right)$ are placed 10 meters from array $(A)$, with angular separation of $60^{\circ}$. (b) Experiment 2. Three arrays $\left(A_{1} \ldots A_{3}\right)$ surround the $20 \times 20 \mathrm{~m}$ sensing region containing 14 receiver nodes $\left(R_{1} \ldots R_{14}\right)$.

For Experiment 1, we perform 50 bearing estimates for each node surrounding the array. The average bearing errors are displayed in Figure 10a. For Experiment 2 , we perform approximately 35 bearing estimates from each anchor to all target nodes, resulting in a total of 105 estimates per target and 1470 estimates total. Figure 10a shows the average error for each bearing from Experiment 1, and the distribution of bearing estimate errors from Experiment 2 are shown in Figure 10b. The average bearing estimation error is $3.2^{\circ}$ overall, with a $6.4^{\circ}$ accuracy at the 90th percentile. The errors from both experiments are consistent with our bearing error analysis in Section 4. 


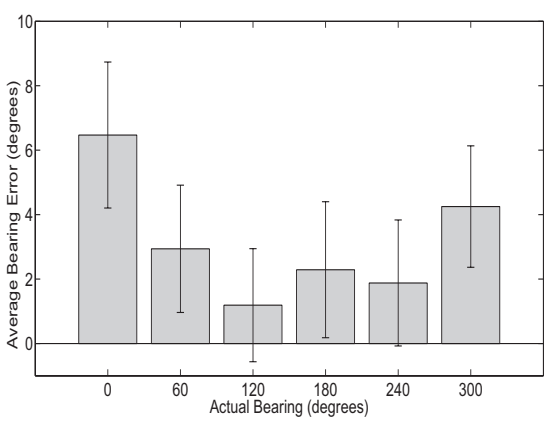

(a)

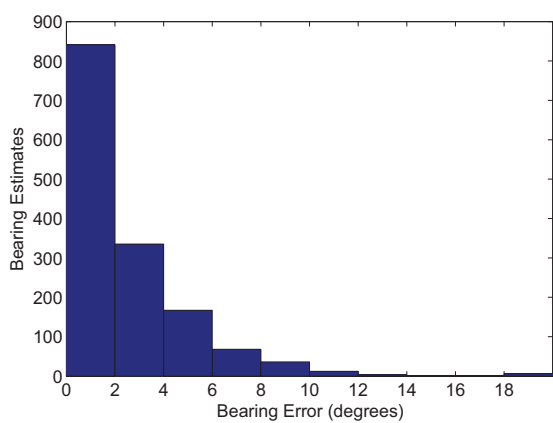

(b)

Fig. 10. Experimental results. (a) Experiment 1 average bearing error with respect to array orientation (sample size of 50). (b) Experiment 2 bearing error distribution (sample size of 1470).

In addition, we evaluate the latency of this method. Because we would like to use this system for mobile sensors in addition to stationary nodes, the array must perform its RIMs as fast as possible so that the sensor has not had a chance to significantly change its position. In order to keep the latency to a minimum, we perform an analysis of the different component execution times. Table 1 lists the execution deadlines of the RIM tasks. These deadlines are enforced via software interrupts and were chosen to give each task enough time to complete, assuming a reasonable amount of jitter.

\begin{tabular}{|l|l|}
\hline Task & Latency $(\mathrm{ms})$ \\
\hline \hline Clock synchronization & 162 \\
\hline Acquire and calibrate radio & 6.48 \\
\hline Transmit / Receive & 63.2 \\
\hline Restore radio driver & 49.91 \\
\hline Report phase & 250 \\
\hline Bearing estimation & 5 \\
\hline
\end{tabular}

Table 1. Latency of bearing estimation tasks.

The array sends one synchronization message and performs two RIMs, for a total time of $401 \mathrm{~ms}$. An additional $255 \mathrm{~ms}$ is required for communication and bearing estimation. Because the target nodes are receivers, no additional latency is incurred by introducing more targets to the sensing region. 


\section{Conclusion}

In this paper, we present a method for rapid distributed bearing estimation in WSNs. The anchor array in our system consists of three nodes, two of which transmit at frequencies that interfere to create a low-frequency beat signal. The phase of this signal is measured by the third node in the array, as well as by multiple target nodes at unknown positions. The phase difference defines a hyperbola, and bearing can be approximated by calculating the angle of the asymptote. Our experimental results show that this technique has an average bearing estimation accuracy of $3.2^{\circ}$, and measurements can be taken in approximately $0.5 \mathrm{sec}$.

Our system is designed to overcome several challenges in WSN AOA determination. The array prototype is easily constructed by fixing three motes together with antennas at orthogonal angles. It is comprised entirely of COTS sensor nodes, and no additional hardware is required because RIM-based ranging only requires use of the radio. Unlike other radio interferometric techniques, our system avoids the modulo $2 \pi$ ambiguity, and therefore the need to perform RIMs on multiple channels, by separating the two transmitting antennas less than half the wavelength of the carrier frequency. Similarly, by constraining the location of one of the RIM receivers to the array, it becomes possible to approximate the bearing of the other receiver without prolonged computation or having to rely on a base station for processing. Our experimental results demonstrate that the accuracy of our prototype implementation is on par with other state-of-the-art AOA techniques.

It is worthwhile noting that this system is designed for eventual use with mobile sensors. Mobility demands rapid localization so that the position estimate is still valid by the time it is computed. Up until now, radio interferometric ranging techniques have been unable to achieve periodic distributed localization at rates fast enough for mobile devices, even slow-moving ones. With this system, we are able to estimate target bearing rapidly enough to support mobile entity localization and navigation. Although at this stage we have not performed tracking or navigation using mobile nodes, we plan on doing so in the near future.

Acknowledgements. This work was supported by ARO MURI grant W911NF06-1-0076, NSF grant CNS-0721604, and NSF CAREER award CNS-0347440. The authors would also like to thank Peter Volgyesi, Metropolitan Nashville Parks and Recreation, and Edwin Warner Park.

\section{References}

1. Niculescu, D., Nath, B.: Ad hoc positioning system (APS) using AOA. In: Proc. of INFOCOM. (2003)

2. Esteves, J., Carvalho, A., Couto, C.: Generalized geometric triangulation algorithm for mobile robot absolute self-localization. In: Proc. of ISIE. (2003)

3. Biswas, P., Aghajan, H., Ye, Y.: Integration of angle of arrival information for multimodal sensor network localization using semidefinite programming. In: 39th Asilomar Conference on Signals, Systems and Computers. (2005) 
4. Ash, J.N., Potter, L.C.: Robust system multiangulation using subspace methods. In: Proc. of IPSN. (2007)

5. Rong, P., Sichitiu, M.: Angle of arrival localization for wireless sensor networks. In: Proc. of SECON. (2006)

6. Nasipuri, A., el Najjar, R.: Experimental evaluation of an angle based indoor localization system. In: 4th International Symposium on Modeling and Optimization in Mobile, Ad Hoc and Wireless Networks. (2006)

7. Chang, H.l., Tian, J.b., Lai, T.T., Chu, H.H., Huang, P.: Spinning beacons for precise indoor localization. In: Proc. ACM SenSys. (2008)

8. Römer, K.: The lighthouse location system for smart dust. In: Proc. of MobiSys. (2003)

9. Ash, J.N., Potter, L.C.: Sensor network localization via received signal strength measurements with directional antennas. In: Proceedings of the Allerton Conference on Communication, Control, Computing. (2004)

10. Friedman, J., Charbiwala, Z., Schmid, T., Cho, Y., Srivastava, M.: Angle-of-arrival assisted radio interferometry (ARI) target localization. In: Proc. of MILCOM. (2008)

11. Maróti, M., Kusý, B., Balogh, G., Völgyesi, P., Nádas, A., Molnár, K., Dóra, S., Lédeczi, A.: Radio interferometric geolocation. In Proc. of ACM SenSys (2005)

12. Capon, J.: High-resolution frequency-wavenumber spectrum analysis. In Proc. of the IEEE 57(8) (1969)

13. Schmidt, R.: Multiple emitter location and signal parameter estimation. IEEE Transactions on Antennas and Propagation 34(3) (1986)

14. Roy, R., Paulraj, A., Kailath, T.: Esprit-a subspace rotation approach to estimation of parameters of cisoids in noise. IEEE Transactions on Acoustics, Speech and Signal Processing 34(5) (1986)

15. Kumaresan, R., Tufts, D.: Estimating the angles of arrival of multiple plane waves. IEEE Transactions on Aerospace and Electronic Systems AES-19(1) (1983)

16. Kushwaha, M., Amundson, I., Volgyesi, P., Ahammad, P., Simon, G., Koutsoukos, X., Ledeczi, A., Sastry, S.: Multi-modal target tracking using heterogeneous sensor networks. In: Proc. of ICCCN. (2008)

17. Priyantha, N.B., Miu, A.K.L., Balakrishnan, H., Teller, S.: The cricket compass for context-aware mobile applications. In: Proc. of MobiCom. (2001)

18. Altun, K., Koku, A.: Evaluation of egocentric navigation methods. In: IEEE International Workshop on Robot and Human Interactive Communication. (2005)

19. Bekris, K.E., Argyros, A.A., Kavraki, L.E.: Angle-based methods for mobile robot navigation: Reaching the entire plane. In: Proc. of ICRA. (2004)

20. Kendig, K.: Conics. Mathematical Association of America (1938)

21. Dutta, P., Grimmer, M., Arora, A., Bibyk, S., Culler, D.: Design of a wireless sensor network platform for detecting rare, random, and ephemeral events. In Proc. of IPSN/SPOTS (2005)

22. Carr, J.: Practical Antenna Handbook, Fourth Edition. McGraw Hill (2001)

23. Kusý, B., Dutta, P., Levis, P., Maróti, M., Lédeczi, A., Culler, D.: Elapsed time on arrival: a simple and versatile primitive for canonical time synchronization services. International Journal of Ad Hoc and Ubiquitous Computing 2(1) (2006) 